\title{
Evidence for rapid variability in the optical light curve of the Type la SN 2014J $\mathrm{J}^{\star \star \star}$
}

\author{
A. Z. Bonanos and P. Boumis
}

\author{
IAASARS, National Observatory of Athens, 15236 Penteli, Greece \\ e-mail: bonanos@astro.noa.gr
}

Received 26 November 2014 / Accepted 25 October 2015

\begin{abstract}
We present results of high-cadence monitoring of the optical light curve of the nearby, Type Ia SN 2014J in M 82, using the $2.3 \mathrm{~m}$ Aristarchos telescope. $B$ and $V$-band photometry on days 15-18 after $t_{\max }(B)$ was obtained with a cadence of 2 min per band, revealing evidence for rapid variability at the 0.02-0.05 mag level on timescales of 15-60 min on all four nights. The decline slope was measured as steeper in the $B$-band than in the $V$-band, and to steadily decrease in both bands from 0.15 mag day $^{-1}$ (night 1 ) to 0.04 mag day $^{-1}$ (night 4) in $V$, and from 0.19 mag day $^{-1}$ (night 1) to 0.06 mag day $^{-1}$ (night 4) in $B$, corresponding to the onset of the secondary maximum. We propose that rapid variability could be due to one or a combination of the following scenarios: the clumpiness of the ejecta, their interaction with circumstellar material, the asymmetry of the explosion, or the mechanism causing the secondary maximum in the near-infrared light curve. We encourage the community to undertake high-cadence monitoring of future, nearby and bright supernovae to investigate the intraday behaviour of their light curves.
\end{abstract}

Key words. supernovae: individual: SN 2014J - supernovae: general - galaxies: individual: M 82

\section{Introduction}

Nearby supernovae offer the opportunity to explore the shorttimescale and low-amplitude variability properties of their light curves. Even though several nearby, bright supernovae ${ }^{1}(\mathrm{SNe})$ have been discovered recently (e.g. the Type Ia SN 2011fe and SN 2013aa, the Type IIP SN 2013ej; Nugent et al. 2011; Waagen 2013; Richmond 2014, respectively), no high-cadence variability search has been undertaken so far to explore variability on timescales of minutes or hours. Typical photometric monitoring of $\mathrm{SNe}$ consists of a single observation per night, or every few nights in several filters. Therefore, the intraday behavior of the light curves of SNe remains uncharted territory ${ }^{2}$.

To rectify the situation, we performed high-cadence photometry of the nearby Type Ia supernova (SN Ia) SN 2014J (see e.g. Foley et al. 2014, and references therein), which was discovered in the galaxy M 82 in January 2014 (Fossey et al. 2014). Reaching $V_{\max }=10.61 \pm 0.05$ mag (Foley et al. 2014), it was ideally suited for a variability search, which we conducted in February 2014 with the $2.3 \mathrm{~m}$ Aristarchos telescope. Just before the submission of this work, Siverd et al. (2015) reported relatively high-cadence photometry of SN 2014J with the $4.2 \mathrm{~cm}$

* Based on observations made with the $2.3 \mathrm{~m}$ Aristarchos telescope, Helmos Observatory, Greece, which is operated by the Institute for Astronomy, Astrophysics, Space Applications and Remote Sensing, National Observatory of Athens, Greece.

$\star \star$ Full Table 2 is only available at the CDS via anonymous ftp to cdsarc.u-strasbg.fr (130.79.128.5) or via

http://cdsarc.u-strasbg.fr/viz-bin/qcat?J/A+A/585/A19

1 http://www . rochesterastronomy . org/snimages/snmag. html

2 While this paper was under review, Olling et al. (2015) reported serendipitous, high-cadence monitoring (every $30 \mathrm{~min}$ ) of three Type Ia supernovae by the Kepler mission.
Kilodegree Extremely Little Telescope North (KELT-N), thereby placing a $4.5 \%(3 \sigma)$ upper limit on short timescale variations. This paper reports the results of our monitoring survey: the observations and data reduction are presented in Sect. 2, the analysis in Sect. 3, the results in Sect. 4, and the discussion and conclusions in Sect. 5.

\section{Observations and data reduction}

SN 2014J was monitored for variability with the LN CCD camera on the $2.3 \mathrm{~m}$ Aristarchos telescope for four consecutive nights: 2014 February 16-19 (nights 1-4 or N1-4), corresponding to days 14.8-18.2 after $t_{\max }(B)=2456690.5 \pm 0.2$ (JD; Foley et al. 2014). The $1024 \times 1024$ SITeAB back-illuminated CCD has pixels that are $24 \mu \mathrm{m}$ in size, which map to $0.28^{\prime \prime}$ pixel $^{-1}$ on the focal plane, making each image $4.8^{\prime}$ on the side. Figure 1 provides a finder chart, labelling the position of the $\mathrm{SN}$ and the comparison stars (S1-S5) used in the analysis below.

SN 2014J was observed for about eight hours per night to obtain differential photometry. Sequences of alternating $V$-band and $B$-band images were obtained with five and $20 \mathrm{~s}$ exposure times, respectively, yielding 400-500 images per band each night and a cadence of two minutes per band. Small gaps in the data are mainly the result of recentering that was performed in between typical sequences of 100 images, because the absence of guiding caused the target to drift during the sequences.

Initial reduction of the images was performed using standard routines in the $\operatorname{IRAF}^{3}$ ccdproc package (i.e. bias level subtraction, flat field division). The images were aligned with the IRAF

3 IRAF is distributed by the National Optical Astronomy Observatory, which is operated by the Association of Universities for Research in Astronomy (AURA) under cooperative agreement with the National Science Foundation. 


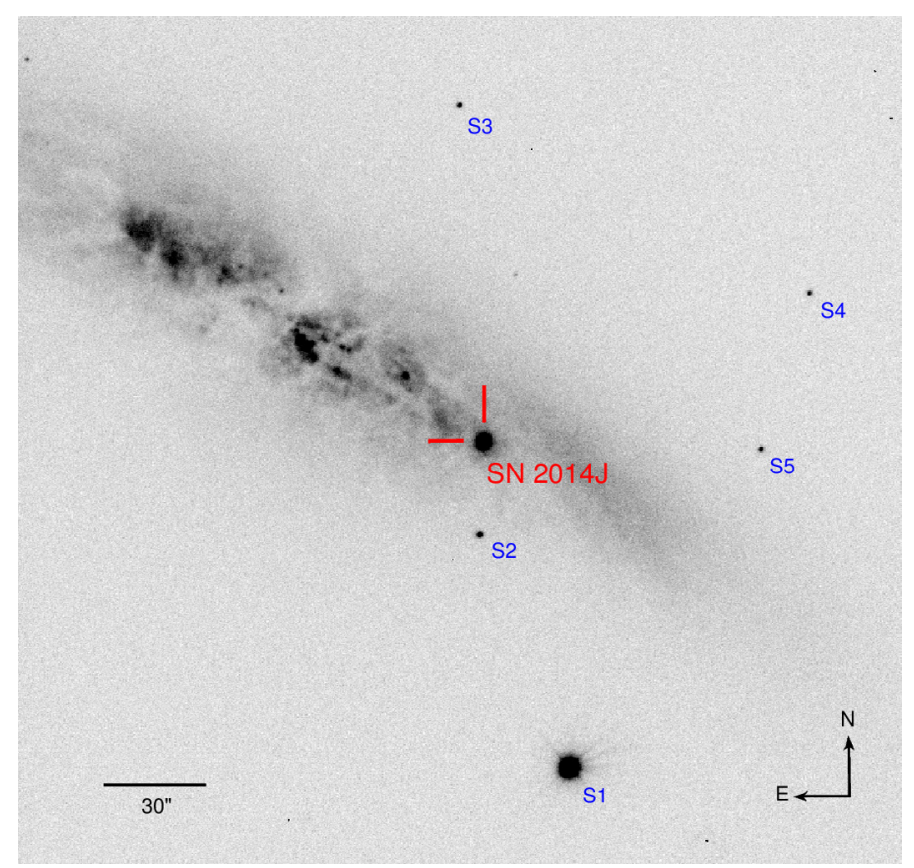

Fig. 1. $V$-band image of SN 2014J in M 82, obtained on 2014 February 16 with the Aristarchos telescope. The positions of the five comparison stars (S1-S5) used in the analysis are labelled.

imalign task, while the seeing was calculated using the IRAF task psfmeasure. The statistical mode values for the seeing in the $V$-band images each night were $1.2^{\prime \prime}, 1.4^{\prime \prime}, 1.2^{\prime \prime}, 1.2^{\prime \prime}$, respectively, and $1.2^{\prime \prime}, 1.6^{\prime \prime}, 1.05^{\prime \prime}, 1.25^{\prime \prime}$ in the $B$-band. Typical $\sigma$ values were $0.55^{\prime \prime}$ for $\mathrm{N} 2$ and $0.2-0.3^{\prime \prime}$ for the other nights. Note that, on $\mathrm{N} 1 / \mathrm{N} 3$, the $\mathrm{S} / \mathrm{N}$ of the maximum pixel value of $\mathrm{S} 1$ in $B$ (190/185) exceeded the mean $\mathrm{S} / \mathrm{N}$ of the $B$-flat (176/172).

\section{Analysis}

Aperture photometry was extracted from the supernova and the five brightest comparison stars available in the field, using a 5-pixel or 1.4" radius with the IRAF apphot package and a 5-pixel wide sky annulus 15 pixels from the source. Typical photometric errors are $1 \mathrm{mmag}$ for the $\mathrm{SN}$ and $\mathrm{S} 1,0.01 \mathrm{mag}$ for S2, and 0.01-0.02 mag for S3-S5.

The instrumental magnitudes for the SN and S1 were transformed to the standard system by computing zeropoint offsets and colour terms, using $B$ and $V$ magnitudes for SN 2014J on N1 from Foley et al. (2014) and for S1 from the APASS Catalogue 4 . The resulting transformation equations are

$V=v-1.320+0.056 \cdot(B-V)$

$B=b-2.609+0.005 \cdot(B-V)$,

where $b$ and $v$ are the instrumental magnitudes.

Differential light curves were created by taking the difference between the instrumental magnitude of the supernova and each of the five comparison stars. S1 yields the smallest scatter, with an error in each point of $1.4 \mathrm{mmag}$. However, due to its brightness, it was saturated or exceeded the non-linearity limit of the CCD ( 45000 ADU) in the best seeing $B$-band images, creating gaps in the differential curves with respect to $S 1$.

\footnotetext{
4 http://www. aavso.org/apass/
}

Furthermore, the absence of guiding caused S1 to intercept a bad column, creating spikes in the light curves in both bands, which were selected visually and removed. Figure 2 presents the differential, calibrated, light curves with respect to $S 1$, which show the expected decline of the $\mathrm{SN}$ and onset of the secondary maximum discussed below.

Figures 3-6 show the differential light curves with respect to all comparison stars available each night. We used a detrending algorithm to reconstruct the light curves, rule out intrinsic variability in $\mathrm{S} 1$ (as no variability information for $\mathrm{S} 1$ is available in the literature), and correct for systematic effects. We applied the Trend Filtering Algorithm (TFA; Kovács et al. 2005), as implemented in the VARTOOLS package (Hartman et al. 2008), using S2-S5 to reconstruct the light curves of the SN and S1. Because S2-S5 are fainter, this method introduced noise at the level of 0.01-0.02 mags to the light curves of the brighter stars, however, we found that the variability signal remains, although it is still affected by systematics. The two lower panels of Figs. 3-6 present the detrended light curves and the differential curve S1$\mathrm{S} 2$, respectively. The latter illustrates systematics present in the photometry, which are discussed in the next section. Features that are present in all light curves (more clearly illustrated in the binned curves) and that correspond to a featureless S1-S2 curve provide evidence for rapid variability in the $\mathrm{SN}$.

We next performed a test using artificial stars to assess the variability signal seen in the differential light curves. We inserted artificial stars in each of the $V$-band images taken on $\mathrm{N} 3$, which displays the largest variability, using the appropriate point spread function derived for each image with the daophot package in IRAF. We inserted three stars at the magnitude of the SN along the disk of the galaxy at locations with both smoother and more complex galaxy background, three stars at the magnitude of S2 at the same distance from the galaxy disk, and three stars at the magnitude and distance of S1. We then performed aperture photometry, as described above, and constructed differential light curves. These were found to be dominated by Poisson noise and not to show any patterns resembling the variability signal. In particular, the standard deviation $(\sigma)$ of the points for the "artificial" $\mathrm{SN}$ in all three positions tested were 4.5-4.8 mmag, while we found $\sigma=1.6 \mathrm{mmag}$ for $\mathrm{SN}-\mathrm{S} 1, \sigma=0.0115 \mathrm{mag}$ for $\mathrm{SN}-\mathrm{S} 2$, and $\sigma=0.012 \mathrm{mag}$ for $\mathrm{S} 1-\mathrm{S} 2$. The artificial star test thus provides additional support for the variability signal originating in the SN.

\section{Results}

The densely sampled differential light curves shown in Figs. 26 provide a measure of the intranight decline rate of SN 2014J. Table 1 presents the decline rates measured each night in $V$ and $B$ based on least-square fits to the differential and detrended light curves shown in Figs. 3-6. The error bars represent the rootmean-square (rms) error of the points to the fit. We note that the slopes inferred for SN-S1 are identical for the calibrated and instrumental light curves. All values are in agreement within errors, except for those derived using $\mathrm{SN}-\mathrm{S} 1$ on $\mathrm{N} 1$, because of the larger gaps in the photometry on that night. Adopting the values derived from $\mathrm{SN}-\mathrm{S} 2, \mathrm{SN} 2014 \mathrm{~J}$ declines with a rate $\alpha$ of $0.15 \pm 0.01,0.09 \pm 0.01,0.07 \pm 0.02,0.04 \pm 0.01$ mag day $^{-1}$ in the $V$-band and $0.19 \pm 0.01,0.12 \pm 0.01,0.13 \pm 0.02$, $0.06 \pm 0.02$ mag day $^{-1}$ in the $B$-band ${ }^{5}$, on N1-4, respectively. We therefore find the $B$-band to fade at a faster rate than the $V$-band, as expected, and the slope in each filter to vary from

\footnotetext{
5 No extinction or atmospheric corrections were applied.
} 


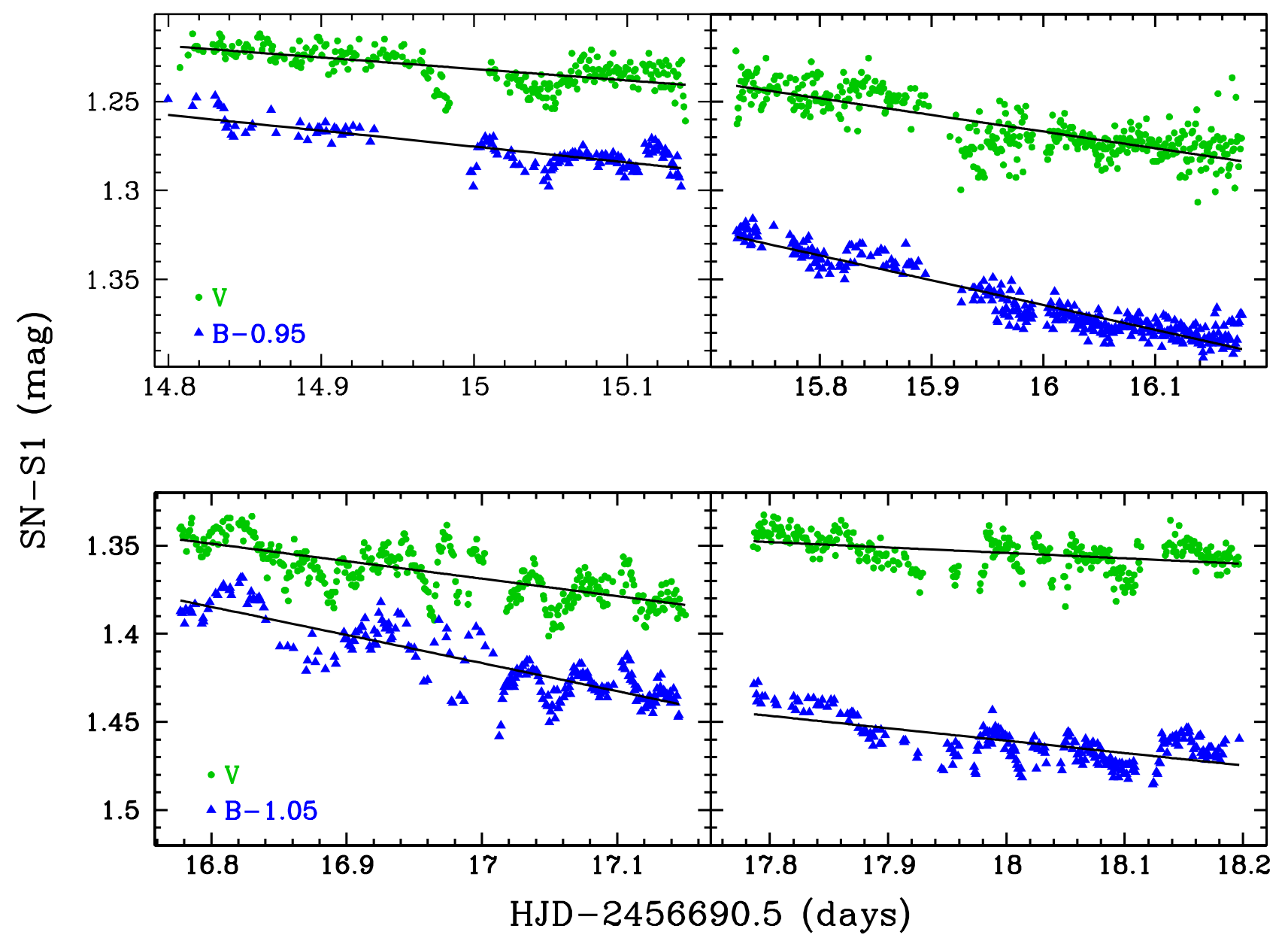

Fig. 2. Calibrated $V$-band (circles) and $B$-band (triangles) differential light curves of SN 2014J, compared with Star 1, as a function of the time in days measured since $t_{\max }(B)=2456690.5$ (JD; Foley et al. 2014). Each panel corresponds to one night of observations. The $B$-band curves are offset by $0.95 \mathrm{mag}$ in the upper panels and $1.05 \mathrm{mag}$ in the lower panels for clarity. The black lines represent least-square fits to the photometry.

night to night. The decrease in the slope, observed in both bands, corresponds to the onset of the secondary maximum in the nearinfrared light curve (Kasen 2006; Jack et al. 2015), which is also seen in the light curves of SN 2014J presented by Foley et al. (2014), Amanullah et al. (2014), Marion et al. (2015), Kawabata et al. (2014), and Ashall et al. (2014).

More importantly, we report evidence for rapid variability in both the $V$ - and $B$-band light curves of SN 2014J. We find that the level of variability varies from night to night and is best traced by the SN-S1 curve, because of the accuracy in the photometric measurements achieved for these bright stars. N3 and $\mathrm{N} 4$ exhibit the largest activity with sinusoidal-like variations of amplitude up to $0.05 \mathrm{mag}$, while variability on other nights is typically at the 0.02-0.03 mag level. A Fourier analysis of the SN-S1 curve did not yield a significant periodicity.

While the precision of our measurements, based on the $1 \sigma$ error bars resulting from the aperture photometry, is at the $1.4 \mathrm{mmag}$ level for SN-S1 (and at the $0.6 \mathrm{mmag}$ level for the binned curve), we must account for correlated sources of error (red noise) to quantify the significance of the variability detection. Sources of correlated error include the changing airmass, drifting of the stars across the CCD and other instrumental parameters. We follow the procedure outlined by Pont et al. (2006), which estimates the amount of correlated noise by calculating the dispersion $\sigma_{N}$ from the binned residuals (after subtracting a best fit model, in our case the decline rate) as a function of
$N$ points and which finds the values for the white $\left(\sigma_{\mathrm{w}}\right)$ and red $\left(\sigma_{\mathrm{r}}\right)$ noise that best fit the equation

$\sigma_{N}^{2}=\frac{\sigma_{\mathrm{w}}^{2}}{N}+\sigma_{\mathrm{r}}^{2}$

The estimated values of $\sigma_{\mathrm{w}}, \sigma_{\mathrm{r}}$, and $\sigma_{N}$ for each night and filter are shown in the last three columns of Table 1, using the SN-S1 data and $N=30(\sim 1 \mathrm{~h})$, which was selected to represent the longest timescale of the detected variability. Note that the values computed with $N=10,20,40$ were very similar. If rapid variability is present, then the $\sigma_{N}$ values are overestimates, as all points of the light curves were used for the calculation. Given the derived $\sigma_{N}$ values (3-6 mmag), we find the 0.02-0.05 mag variations to be statistically significant. At face value, the $0.05 \mathrm{mag}$ variation in N3 (at HJD $=2456707.46$ 2456707.48 ) has a $8.2 \sigma$ significance in $V$ and $10 \sigma$ in $B$, and the 0.04 mag variation in $\mathrm{N} 4$ (at HJD $=2456708.48-2456708.52$ ) is a $7.7 \sigma$ detection in $V$, and a $6.3 \sigma$ detection in $B$. In $\mathrm{N} 2$, the 0.02 mag variation at $\mathrm{HJD}=2456706.32$ is significant at the $3.8 \sigma$ level in $V$ and $5.6 \sigma$ in $B$, while in N1, the 0.03 mag variation (at $\mathrm{HJD}=2456705.47-2456705.51$ ) is at the $6.2 \sigma(V)$ and $9.7 \sigma(B)$ level. The timescale of sinusoidal-like variations ranges from 15-60 min. On N3, the $B$-band is found to precede the $V$-band by $\sim 10 \mathrm{~min}$. The $B-V$ colour gradually increases within each night, displaying an rms scatter of about $0.01 \mathrm{mag}$. 


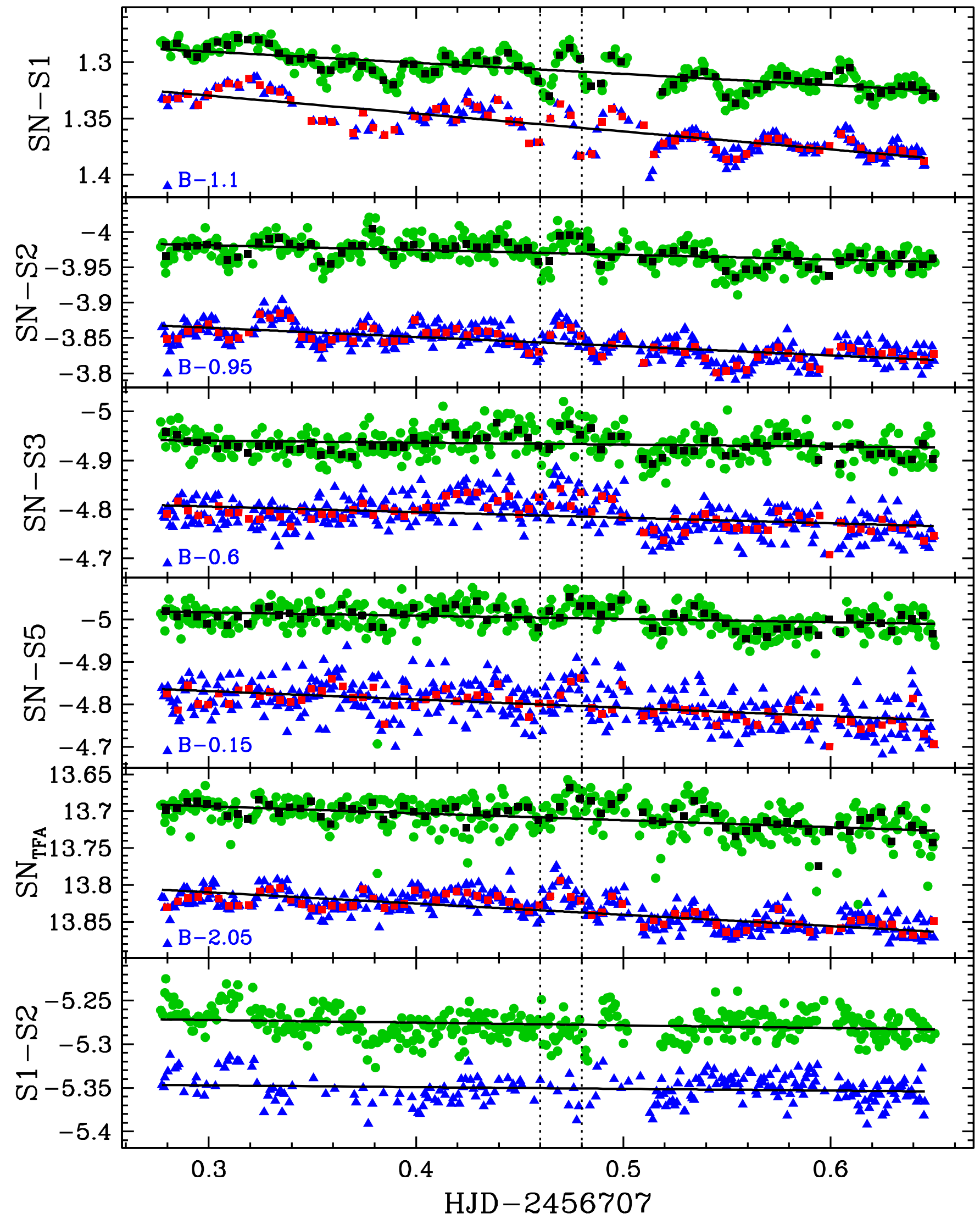

Fig. 3. Instrumental $B$-band (blue triangles) and $V$-band (green circles) light curves of SN 2014J obtained on N3. The first four panels show the differential light curves of the SN with respect to S1-S3 and S5, respectively, followed by a panel showing the reconstructed light curves using the trend-fitting algorithm (TFA) and the differential curve S1-S2. Solid lines correspond to least-square fits to the data, while black and red squares correspond to the binned $V$ - and $B$-band light curve, respectively, using a bin size of 0.005 days. The $B$-band curves are offset for clarity by the amount indicated in each panel. Dotted lines indicate regions of significant variability. 


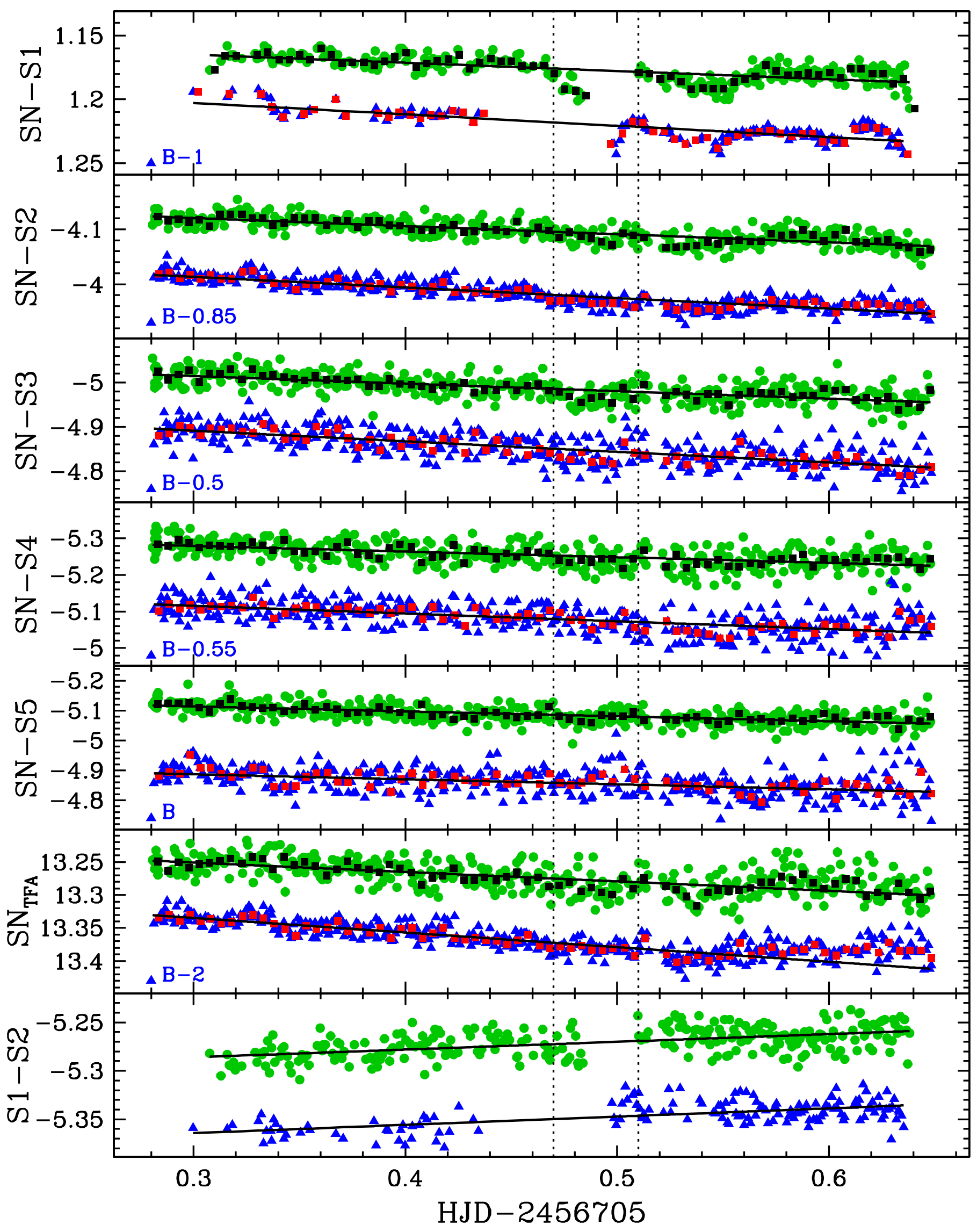

Fig. 4. Same as Fig. 3, but for N1. On this night all five comparison stars were available, therefore the first five panels show the differential light curves with respect to S1-S5, respectively, followed by a panel showing the reconstructed light curves using the trend-fitting algorithm and the differential curve S1-S2. 


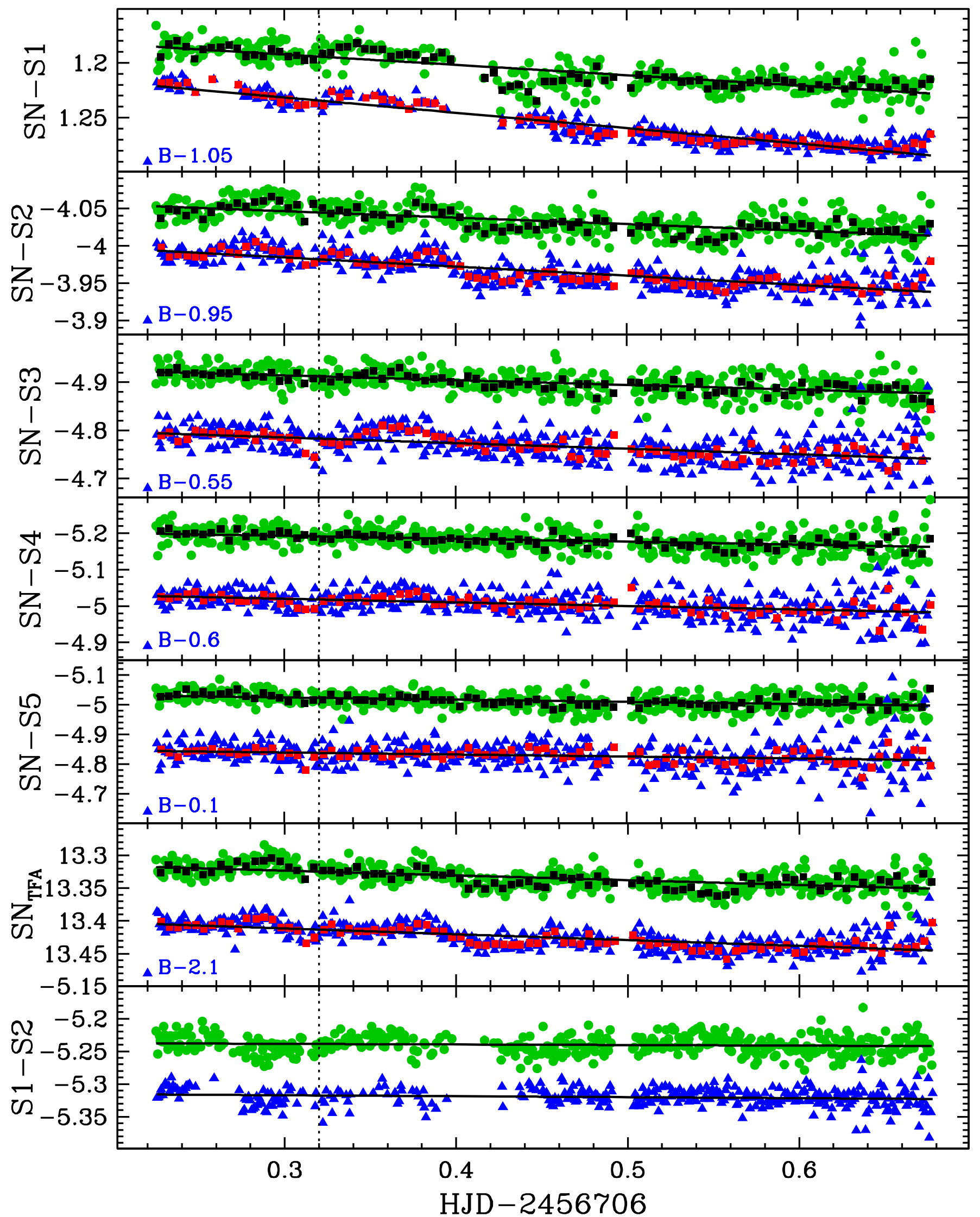

Fig. 5. Same as Fig. 4, but for N2. 


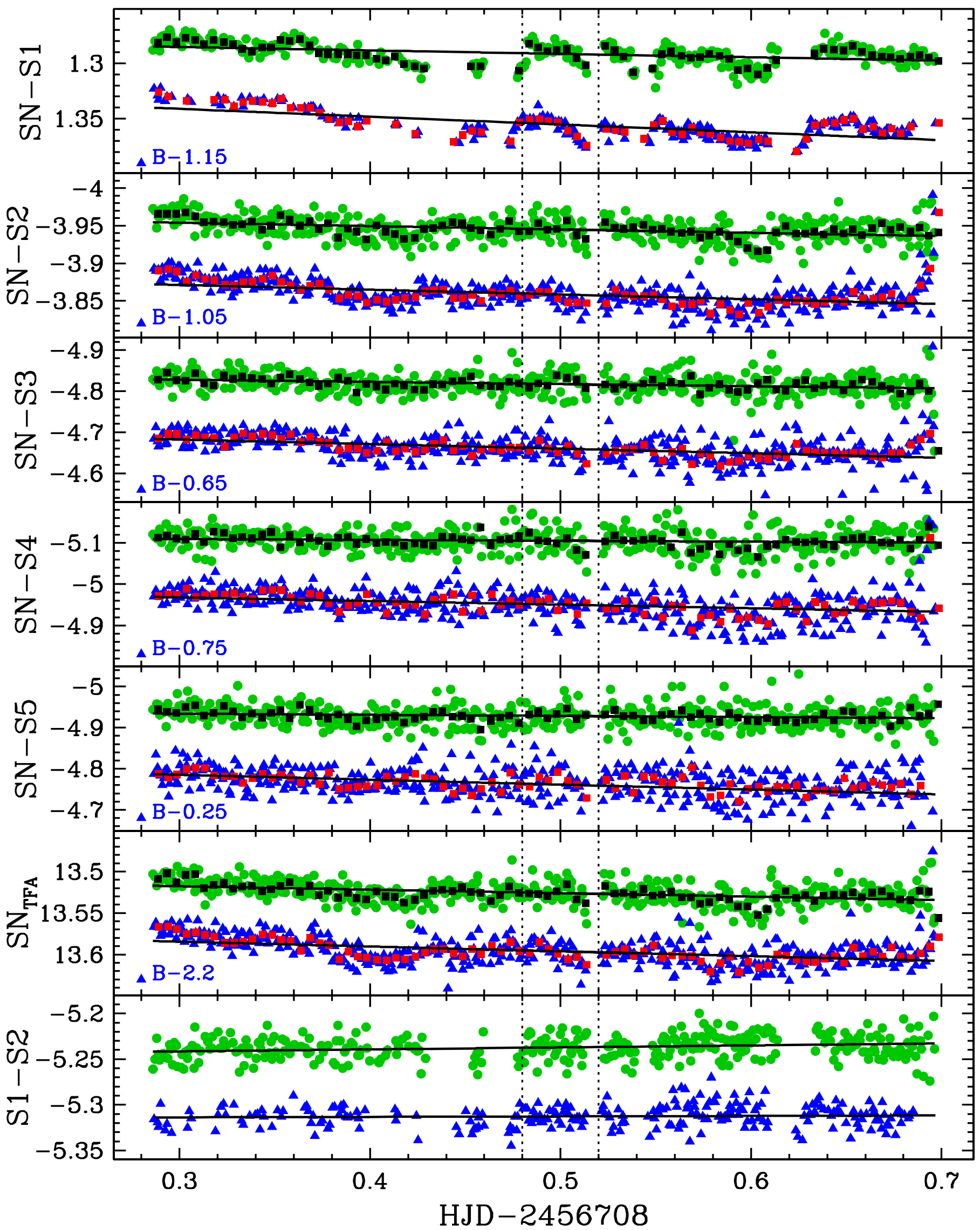

Fig. 6. Same as Fig. 4, but for N4. 
A\&A 585, A19 (2016)

Table 1. Decline rates $\alpha\left(\operatorname{mag~day~}^{-1}\right)$ and values of $\sigma_{\mathrm{w}}, \sigma_{\mathrm{r}}, \sigma_{N}(\mathrm{mag})$ based on SN-S1 of the $V$ - and $B$-band light curves of SN 2014J.

\begin{tabular}{|c|c|c|c|c|c|c|c|c|c|c|}
\hline Night & Filter & $\alpha_{\mathrm{SN}-\mathrm{S} 1}$ & $\alpha_{\mathrm{SN}-\mathrm{S} 2}$ & $\alpha_{\mathrm{SN}-\mathrm{S} 3}$ & $\alpha_{\mathrm{SN}-\mathrm{S} 4}$ & $\alpha_{\mathrm{SN}-\mathrm{S} 5}$ & $\alpha_{S N_{\mathrm{TFA}}}$ & $\sigma_{\mathrm{w}}$ & $\sigma_{\mathrm{r}}$ & $\sigma_{N}$ \\
\hline 1 & $\bar{V}$ & $0.06 \pm 0.01$ & $0.15 \pm 0.01$ & $0.17 \pm 0.02$ & $0.15 \pm 0.03$ & $0.16 \pm 0.02$ & $0.14 \pm 0.02$ & 0.0086 & 0.0045 & 0.0048 \\
\hline 2 & V & $0.09 \pm 0.01$ & $0.09 \pm 0.01$ & $0.10 \pm 0.02$ & $0.08 \pm 0.03$ & $0.07 \pm 0.03$ & $0.07 \pm 0.01$ & 0.0107 & 0.0050 & 0.0053 \\
\hline 3 & V & 0.01 & $0.07 \pm 0.02$ & $0.04 \pm 0.03$ & & $0.08 \pm 0.03$ & $0.09 \pm 0.04$ & 0.0 & 0.0052 & 0.0061 \\
\hline 4 & V & $03 \pm 0.01$ & $0.04 \pm 0.01$ & $0.05 \pm 0.02$ & $0.02 \pm 0.03$ & $0.03 \pm 0.02$ & $0.04 \pm 0.01$ & 0.0131 & 0.0046 & 0.0052 \\
\hline 1 & $\bar{B}$ & $0.09 \pm 0.01$ & $0.19 \pm 0.01$ & $0.24 \pm 0.03$ & $0.21 \pm 0.03$ & $0.17 \pm 0.05$ & $0.22 \pm 0.05$ & 0.0104 & 0.0025 & 0.0031 \\
\hline 2 & $B$ & 01 & $0.12=$ & $0.12 \pm 0.03$ & $0.10 \pm 0.03$ & $0.07 \pm$ & $0.09 \pm$ & 0.0074 & 0.0033 & 0.0036 \\
\hline 3 & $B$ & $0.16 \pm 0.01$ & $0.13 \pm 0.02$ & $0.11 \pm 0.03$ & $\ldots$ & $0.20 \pm 0.04$ & $0.15 \pm 0.03$ & 0.0186 & 0.0036 & 0.0050 \\
\hline 4 & $B$ & $0.07 \pm 0.01$ & $0.06 \pm 0.02$ & $0.11 \pm 0.03$ & $0.09 \pm 0.04$ & $0.12 \pm 0.04$ & $0.06 \pm 0.01$ & 0.0128 & 0.0059 & 0.0063 \\
\hline
\end{tabular}

Table 2. Calibrated differential photometry of SN 2014J compared to $\mathrm{S} 1$ in the $V$ and $B$-bands.

\begin{tabular}{lccc}
\hline \hline HJD-2 450 000 & Filter & SN-S1 & $\sigma_{\text {SN-S1 }}$ \\
\hline 6705.30817 & $V$ & 1.231 & 0.001 \\
6705.31330 & $V$ & 1.224 & 0.001 \\
6705.31679 & $V$ & 1.212 & 0.001 \\
6705.31766 & $V$ & 1.220 & 0.001 \\
6705.31853 & $V$ & 1.219 & 0.001 \\
\hline
\end{tabular}

Notes. The full table is available at the CDS.

\section{Discussion and conclusions}

The high-cadence, high-precision photometry obtained with the $2.3 \mathrm{~m}$ Aristarchos telescope was crucial in providing evidence for rapid variability in the optical light curve of SN 2014J. A cadence of $30 \mathrm{~min}$, such as that of the Kepler Mission, would not have been sufficient to detect the sinusoidal-like variations nor any variability pattern seen with the 2 -min cadence of our observations. At face value, our photometric precision of 3-6 mmag (based on the estimation of red noise) or 2 mmag (based on artificial star tests on N3) for SN-S1 allow for statistically significant detections of variability at the level of 0.02-0.05 mag on all nights, peaking on N3. This implies that the phenomenon is common or perhaps even ubiquitous in SNe, unless it is produced by a mechanism related exclusively to the onset of the secondary maximum

Theoretical models of SN light curves (e.g. Kasen et al. 2006; Sim et al. 2013; Fink et al. 2014) do not make predictions on such short time scales and therefore cannot be compared with the photometry presented here. We propose one or a combination of the following scenarios for the origin of the variability: (i) clumping of the ejecta, possibly caused by structures of intermediate mass elements in the outer layers (Hole et al. 2010); (ii) interaction of the ejecta with circumstellar material, inferred by Foley et al. (2014); (iii) asymmetry of the ejecta, as the explosion is not expected to be spherically symmetric (as inferred from spectropolarimetry, e.g. see review by Wang \& Wheeler 2008); (iv) the onset of the secondary maximum, which corresponds to a sudden decrease in the flux mean opacity caused by the transition from doubly to singly ionized iron group elements (see e.g. Pinto \& Eastman 2000).

Using the photospheric velocity $\sim$ two weeks after maximum $\left(-10,600 \mathrm{~km} \mathrm{~s}^{-1}\right.$; estimated from Fig. 15 of Foley et al. 2014), we estimate the $\mathrm{SN}$ to have a radius of $104 \mathrm{AU}$ on day 17. The 0.02-0.05 mag variability corresponds to a fractional change of $\sim 2-5 \%$ in magnitude and flux, respectively, or a $\sim 1-2.5 \%$ fractional change in radius, assuming constant luminosity, and that the variation is due to a change of radius, i.e. 1-2.6 AU. The observed fluctuation, however, is an average variation of flux over the projected surface of the young remnant; the 1-2.6 AU radius change gives an estimate of the surface area fluctuating. Siverd et al. (2015) report relatively high-cadence photometry of SN 2014J with the $4.2 \mathrm{~cm}$ Kilodegree Extremely Little Telescope North (KELT-N), thereby placing a $4.5 \%(3 \sigma)$ upper limit on short timescale variations. The evidence for variability at the level of $2-5 \%$ presented in this work is therefore consistent with the results of the measurement with KELT-N.

In conclusion, we strongly encourage the community to undertake high-cadence and high-precision monitoring campaigns of future, nearby, and bright supernovae to (i) confirm the presence of rapid variability; (ii) determine whether it occurs in both $\mathrm{SNe}$ Ia and II light curves; and (iii) differentiate between the scenarios for its origin. If variability is due to asphericity in the explosion, it will provide evidence for distinguishing the nature of SNe Ia progenitors (see Livio \& Pringle 2011). Furthermore, light-curve variability, if shown to be ubiquitous, is likely to contribute to the scatter in the mean magnitude of SN Ia calibrators, which remains one of the largest factors in the uncertainty of the Hubble constant (e.g. Riess et al. 2011). In any case, we expect rapid variability to provide a new window into the physics of supernovae.

Acknowledgements. The authors thank the anonymous referees for constructive comments that helped clarify and improve the presentation of our results. The data are available upon request. A.Z.B. acknowledges helpful discussions with Saurabh Jha, Stephen Williams, Kris Stanek, and Mercedes López-Morales during the preparation of this manuscript. The authors acknowledge use of the IDL implementation of the red noise calculation by J. Rogers and the excellent support of John Alikakos during the observations. This research has made use of NASA's Astrophysics Data System.

\section{References}

Amanullah, R., Goobar, A., Johansson, J., et al. 2014, ApJ, 788, L21 Ashall, C., Mazzali, P., Bersier, D., et al. 2014, MNRAS, 445, 4427 Fink, M., Kromer, M., Seitenzahl, I. R., et al. 2014, MNRAS, 438, 1762 Foley, R. J., Fox, O., McCully, C., et al. 2014, MNRAS, 443, 2887 Fossey, J., Cooke, B., Pollack, G., Wilde, M., \& Wright, T. 2014, Central Bureau Electronic Telegrams, 3792, 1

Hartman, J. D., Gaudi, B. S., Holman, M. J., et al. 2008, ApJ, 675, 1254

Hole, K. T., Kasen, D., \& Nordsieck, K. H. 2010, ApJ, 720, 1500

Jack, D., Baron, E., \& Hauschildt, P. H. 2015, MNRAS, 449, 3581

Kasen, D. 2006, ApJ, 649, 939

Kasen, D., Thomas, R. C., \& Nugent, P. 2006, ApJ, 651, 366

Kawabata, K. S., Akitaya, H., Yamanaka, M., et al. 2014, ApJ, 795, L4

Kovács, G., Bakos, G., \& Noyes, R. W. 2005, MNRAS, 356, 557

Livio, M., \& Pringle, J. E. 2011, ApJ, 740, L18

Marion, G. H., Sand, D. J., Hsiao, E. Y., et al. 2015, ApJ, 798, 39

Nugent, P. E., Sullivan, M., Cenko, S. B., et al. 2011, Nature, 480, 344

Olling, R. P., Mushotzky, R., Shaya, E. J., et al. 2015, Nature, 521, 332

Pinto, P. A., \& Eastman, R. G. 2000, ApJ, 530, 757

Pont, F., Zucker, S., \& Queloz, D. 2006, MNRAS, 373, 231

Richmond, M. W. 2014, J. Am. Assoc. Variable Star Observers (JAAVSO), 42, 333

Riess, A. G., Macri, L., Casertano, S., et al. 2011, ApJ, 730, 119

Sim, S. A., Seitenzahl, I. R., Kromer, M., et al. 2013, MNRAS, 436, 333

Siverd, R. J., Goobar, A., Stassun, K. G., \& Pepper, J. 2015, ApJ, 799, 105

Waagen, E. O. 2013, AAVSO Alert Notice, 479, 1

Wang, L., \& Wheeler, J. C. 2008, ARA\&A, 46, 433 